\title{
Treatment outcome and impact of leprosy elimination campaign in Sokoto and Zamfara states, Nigeria
}

\author{
B. E. EBENSO*, S. M. TURETA*** \& S. O. UDO** \\ *The Leprosy Mission, PMB 179, Minna, Niger State, Nigeria \\ **The Leprosy Mission, PMB 01048, Gusau, Zamfara State, Nigeria \\ ***Leprosy Control Programme, MOH, PMB 2113, Sokoto, Sokoto \\ State, Nigeria
}

Accepted for publication 28 March 2001

\begin{abstract}
Summary A Leprosy Elimination Campaign (LEC) was implemented in 37 districts of Sokoto and Zamfara states, Nigeria from 13 August to 30 November 1998. The campaign utilized intensive community mobilization and training of local health personnel to detect hidden leprosy cases. During 8 weeks of case finding, 160,127 persons were screened; 353 new cases of leprosy were detected and placed on MDT; $236(67 \%)$ of new cases detected were classified as MB, 64 cases $(18 \%)$ suffered visible deformities and 24 patients $(6.8 \%)$ were children. Follow-up in December 1999 of patients placed on MDT revealed 97\% PB and 96\% MB cure rates, respectively. Detection of cases in communities led some community leaders to ask for repeat surveys in their communities. Repeat surveys continue to yield new cases. The authors recommend that LECs be maintained for 3 years to accelerate leprosy elimination in the region. The cost effectiveness and impact of LEC in Sokoto-Zamfara are discussed.
\end{abstract}

\section{Introduction}

In 1995, the World Health Organization (WHO) introduced a new initiative, leprosy elimination campaigns (LECs), the purpose of which was to detect and treat hidden leprosy patients. Between 1995 and 1999, LECs have been carried out in 24 endemic countries, ${ }^{1}$ including Nigeria. In July 1999, a WHO consultative meeting assessed, among other parameters, the impact of LECs. ${ }^{2}$ A review of LECs in the 24 countries observed that $>500,000$ new cases had been detected by LECs. Apart from detecting new cases, some LECs were successful in promoting community awareness, reducing stigma, and improving the accessibility of multidrug therapy (MDT) and skills of general health workers for diagnosis and treatment. The meeting also observed, however, that case holding was weak in some LECs and defaulter rates were high, with low treatment completion rates. 
While reports describing the conduct of LECs are increasing, ${ }^{3,4}$ these reports are limited to the objectives of LECs, activities carried out, problems encountered and the immediate gains of LECs namely; i) population covered, ii) health workers/volunteers trained, iii) active cases detected and put on MDT, and iv) new MDT clinics opened during the campaigns. However, reports of cure rates among new patients put on MDT during LECs are few. This paper presents treatment outcome of patients detected during LEC and discusses the cost effectiveness and impact of LEC in Sokoto-Zamfara states, Nigeria.

\section{Background}

Sokoto and Zamfara states situated in the Northwest corner of Nigeria, have a total area of about $68,000 \mathrm{~km}^{2}$ and population of $5.4 \times 10^{6}$ people (1991 census) living in 37 administrative districts (23 in Sokoto and 14 in Zamfara). Over $80 \%$ of the population are Hausa speaking, Moslem rural farmers. There are 625 health facilities in Sokoto-Zamfara region; $116(18.6 \%)$ of these offer MDT services to leprosy patients.

Leprosy work in Sokoto-Zamfara started in 1938 when Sudan Interior Mission built a leprosarium in Sokoto town to cater for approximately 36,000 patients in old Sokoto province. Satellite dispensaries were established in the late 1940s to serve patients who lived far from the leprosarium. Between 1948 and 1992, the leprosy service in the region used dapsone monotherapy as the strategy for control. The introduction of MDT for treatment of leprosy in the region was delayed because Sokoto-Zamfara did not commence a statewide leprosy control programme until 1993 when The Leprosy Mission (TLM) was invited to support implementation of MDT services. Sokoto-Zamfara introduced MDT in October 1993 following a statewide case verification exercise when the registered prevalence was 7142 cases. There was a 96\% reduction in registered cases following MDT implementation to only 273 cases in September 1998. Despite the drastic fall in prevalence, the annual new case detection averages 360 cases, and MB proportion and grade 2 disability rate among new cases remain high. Table 1 shows leprosy control indicators from 1993 to 2000.

The high MB proportion and grade 2 disability rate among new cases from 1993-1997 led to the suspicion of a large number of hidden cases in the region. A LEC was therefore proposed to verify above suspicion and to accelerate elimination activities by detecting hidden cases, and complement routine control activities.

Table 1. Annual leprosy control indicators in Sokoto-Zamfara (1993-2000)

\begin{tabular}{lrrrrrrrrr}
\hline Indicator & 1993 & 1994 & 1995 & 1996 & $\begin{array}{c}1997 \\
\text { Jan-Sept }\end{array}$ & $\begin{array}{r}1998^{\mathrm{a}} \\
(\text { LEC) }\end{array}$ & $1998^{\mathrm{a}}$ & 1999 & 2000 \\
\hline Registered prevalence & 7142 & 2414 & 2240 & 647 & 519 & 273 & - & 266 & 255 \\
New case detection & 158 & 241 & 594 & 236 & 471 & 226 & 353 & 256 & 271 \\
MB proportion (\%) & 65 & 52 & 44 & 52 & 75 & 73 & 67 & 78 & 93 \\
Grade 2 disability (\%) & 34 & 15 & 6 & 11 & 31 & 20 & 18 & 16 & 14 \\
Child proportion (\%) & 5 & 3 & 2 & 8 & 7 & 9 & 6.8 & 6 & 9 \\
\hline
\end{tabular}

\footnotetext{
${ }^{a} 1998$ figures represent the periods January to September (before LEC) and during LEC respectively.
} 


\section{Materials and methods}

Sokoto-Zamfara region was selected for LEC due to (i) high MB and grade 2 disability rates among new cases leading to the suspicion of a large number of hidden patients in the communities, (ii) easy accessibility of majority of communities and (iii) availability of MDT services in the region. A statewide LEC was conducted in the region from 13 August to 30 November 1998 to detect and treat hidden cases using intensive population mobilization (via radio, town criers, and WHO posters to increase awareness of leprosy) and task-oriented training of health staff. This campaign was described by Sofola, in a recent paper, ${ }^{5}$ stating the objectives of and activities carried out during the campaign and immediate achievements of the campaign namely numbers of (i) villages/population covered, (ii) health workers/ volunteers trained, (iii) active cases detected and put on MDT, and (iv) new MDT clinics opened during the campaigns. Except for (iii) above, the foregoing is not repeated here.

Radio messages were broadcast at prime time twice a day for 8 weeks to emphasize curability of leprosy, availability of MDT at no cost to patients and duration of LEC. More than 5000 posters affixed conspicuously at health centres and public places displayed skin lesions of leprosy and invited persons with similar lesions to report to health facilities nearest to them for confirmation. Town criers announced venues and dates of surveys in communities. Announcements by town criers were done 2 days prior to surveys and continued daily throughout the duration of the surveys.

Skin surveys took place in health facilities in selected communities for 8 weeks (4 October to 30 November). Thirteen special teams conducted the screening. Each team comprised a Leprosy Supervisor (LS), a General Health Worker (GHW), and a Voluntary Village Health Worker (VVHW). To ensure quality control of diagnosis, (i) special teams were headed by LS with more than 5 years experience in leprosy control, and (ii) active leprosy cases and suspect cases referred by the teams were re-examined by a monitoring team, of two state leprosy control officers and a physiotherapist, with more than 7 years experience in leprosy control. Cases confirmed by the monitoring team were placed on MDT immediately. During the campaign, cure was defined as completion of fixed duration WHO-MDT. ${ }^{6}$ PB patients were required to take six doses of PB.MDT blister calendar packs within 9 months. MB cases were to take 12 doses of MB.MDT blister calendar packs within 18 months before they were released from treatment (RFT). A review of all patients put on MDT was carried out 1 year after the LEC exercise (in December 1999) to assess the outcome of treatment.

\section{Results}

During 8 weeks of case finding, 160,127 people (107,285 males and 52,842 females) were examined; and 353 new leprosy cases were detected (a rate of 22 cases/10,000 population) as against 226 cases detected by routine control activities (a rate of $0.42 / 10,000$ people, using $5.4 \times 10^{6}$ total population) between January and September 1998. Furthermore, 108 suspect cases were referred by special teams for confirmation, $88(81.5 \%)$ of which suffered fungal infection. The remaining 20 suspect cases (18.5\%) had birthmarks, psoriasis or vitiligo. In addition, the monitoring team discovered 11 cases $(3.1 \%)$ of over diagnosis of burnt-out $\mathrm{MB}$ leprosy and removed them from the register. The number of cases of under-diagnosis cannot be ascertained, as those patients were sent away without the diagnosis being made and no records of under-diagnosis were kept. 
Table 2. LEC in Sokoto-Zamfara. New cases detected by classification, disability grade, and age

\begin{tabular}{lrrcccc}
\hline State & $\begin{array}{c}\text { Population } \\
\text { screened }\end{array}$ & & New cases & & $\begin{array}{c}\text { Disability } \\
\text { grade 2 }\end{array}$ & $<15$ years \\
\hline Sokoto & & PB & MB & Total & & \\
Zamfara & 56,117 & 86 & 141 & 227 & 37 & 12 \\
Total & 104,010 & 31 & 95 & 126 & 27 & 12 \\
& 160,127 & 117 & 236 & 353 & 64 & 24 \\
\hline
\end{tabular}

Table 2 shows $236(67 \%)$ of the 353 leprosy cases were classified as MB, 24 cases $(6.8 \%)$ were $<15$ years of age and 64 patients $(18 \%)$ suffered visible deformities. Table 3 shows 227 (96\%) of MB and 113 (97\%) of PB patients completed fixed duration MDT; and four patients died. All $227 \mathrm{MB}$ patients completed their course of MDT within 12 months and were released from treatment without the need to extend the period of treatment to 18 months. A total of US\$19,712 was spent on LEC in the 37 districts of the region. Table 4 shows comparison of cost of LEC with cost of routine case finding.

\section{Discussion}

The success of the multi-media campaign at prompting a positive health seeking behaviour in 160,127 people is noteworthy. Furthermore, the authors who were sceptical of detecting any more than 200 new cases through LEC, were surprised by the enthusiasm displayed by LS, GHW, VVHW and community leaders during the campaign and at the detection of 353 cases (22/10,000 population). The campaign therefore detected $127(36 \%)$ more cases in 8 weeks than 226 cases $(0.42 / 10,000)$ detected in 36 weeks by routine control activities. Even though it was difficult to ascertain the number of people reached or examined by routine control activities due to poor records, the authors infer from the foregoing that routine control activities are slow at increasing awareness about leprosy and could be reaching only a small proportion of the population. This may perhaps explain why patients hide in the community or report with visible deformity.

Table 3. Post-LEC treatment outcome. December 1999

\begin{tabular}{lcc}
\hline Category & PB (\%) & MB (\%) \\
\hline New cases detected & $117(100)$ & $236(100)$ \\
Released from treatment (cured) & $113(96.6)$ & $227(96.2)$ \\
Died $^{\mathrm{a}}$ & $1(0.85)$ & $3(1.3)$ \\
Transferred out $_{\text {Lost to control }}$ & $1(0.85)$ & $2(0.85)$ \\
& $2(1.7)$ & $4(1.7)$ \\
\hline
\end{tabular}

${ }^{\mathrm{a}} \mathrm{T}$ wo MB patients died of severe lepra reaction and one $\mathrm{MB}$ and one $\mathrm{PB}$ patient died of non-leprosy related causes. 
Table 4. Comparison of cost of routine case finding with cost of LEC

\begin{tabular}{lcc}
\hline & \multicolumn{2}{c}{ Amount spent on activity (US\$) } \\
\cline { 2 - 3 } Activities performed & Routine case finding & Through LEC \\
\hline Salaries & 3252 & 11,120 \\
Training & 1925 & 4830 \\
Travel & 6655 & 1338 \\
Public enlightenment & 1710 & 2067 \\
Administration & 276 & 357 \\
Total & 13,818 & 19,712 \\
& & \\
\hline
\end{tabular}

BENEFITS OF LEC

In addition to promoting public awareness, this campaign combined (i) capacity building of GHW and VVHW to diagnose leprosy, (ii) mobilization of community and health services participation in LEC, (iii) screening of 160,127 people, and (iv) opening of new MDT clinics 5 into one co-ordinated activity in an effective manner and with important interactions. ${ }^{7}$ These aforementioned activities are of ten conducted separately or at different times during routine control activities. Following community mobilization during LEC, awareness has been raised to the extent that active cases continue to report to clinics several months after LEC, claiming they were motivated by messages broadcast during LEC.

\section{COST OF LEC COMPARED WITH ROUTINE CASE FINDING}

The comparison of cost of routine activities with the cost of LEC is interpreted with caution, as it is difficult to ascertain the number of people reached or examined during routine (opportunistic) case finding. However, if number of cases detected is used as the basis of comparison, the cost of LEC screening was US\$19.712 and cost of routine screening in 1998 was US $\$ 13,818$. This means that LEC detected 353 cases at the cost of US\$56 per case while routine screening detected 226 cases at the cost of US\$61 per case.

\section{COST-EFFECTIVENESS OF LEC}

The main criteria for conducting LEC in the region (high MB and grade 2 disability rates among new cases and suspicion of hidden cases), led to intensive multi-media campaign, which encouraged persons with skin lesions to present for examination. The strategy of sensitizing the whole population while inviting only persons with skin lesions for examination made screening for new cases less cumbersome than house-to-house survey, which is of ten very expensive ${ }^{8}$ and would have aimed at examining $5.4 \times 10^{6}$ people in the region. The authors therefore consider the detection of 353 cases from 160,127 people $(22 / 10,000$ population) in 8 weeks cost-effective when compared with 226 cases detected from a population of $5.4 \times 10^{6}(0.42 / 10,000)$ in 36 weeks by routine control activities. The benefits of LEC above are worth the cost especially as LEC was conducted as one activity within a short period. It would hence be justified to continue the media campaign to ensure that everyone learns about signs of leprosy and its cure. 


\section{TREATMENT OUTCOME}

Though case holding was given high priority in this LEC, the high cure rates recorded above were a pleasant surprise to control staff, especially the authors, who perhaps did not expect cure rates higher than the known rates of $81 \%$ in $\mathrm{MB}$ and $92 \%$ in $\mathrm{PB}$ patients in the region (ILEP B report, 1998). This higher than expected cure rates could be attributed to (i) the commitment of LS and GHW to follow-up absentees, (ii) effective health information on compliance to treatment given to patients and (iii) patients' trust in the health service. The cure rates from LEC in Sokoto-Zamfara are higher than MB cure range of 30-89\% and compare favourably with PB cure range of $65-100 \%$ from Indonesia, Nepal, and Philippines. ${ }^{9}$

IMPACT OF LEC

\section{Follow-up LECs}

The detection of 353 patients during LEC compared to 226 patients detected between January and September of the same year, 1998, confirms suspicion of hidden cases in the region and led to the adoption, in the year 2000, of modified LEC campaigns ${ }^{5}$ in other north western states supported by TLM in the hope of detecting all hidden cases. To date, all five states have conducted mini-LECs in a total of 29 districts. Preliminary results show that mini-LECs detected 260 hidden cases within 5 days in 29 districts surveyed (Mini-LEC reports 2000; Abuja, Kebbi, Kogi, Kwara and Niger states). Three of the five states that implemented miniLECs in 2000 plan to extend the exercise to more districts in 2001.

\section{Increased community awareness and participation in leprosy control}

Detection of cases in communities led some community leaders to ask for repeat surveys in their communities (Quarterly Statistical Report 1999, Zamfara state). Repeat surveys continue to yield new cases. Referrals of leprosy from communities to the health service are also increasing (Quarterly Statistical Report 1999, Sokoto state) proving that community awareness remains high. These repeat surveys may contribute to annual new case detection for 1999 and 2000 (256 and 271 cases, respectively) remaining higher than pre-LEC detection figure of 226 in 1998 (see Table 1), suggesting that though multi-media campaigns are effective, not every one has been reached and that there are active cases still hiding in the communities. The campaigns should be sustained to motivate hidden cases to continue to report for confirmation and treatment, if a declining trend in new case detection is to be seen in future.

In addition to strengthening routine activities in the field by training local health workers, detecting hidden cases and increasing awareness of leprosy in the community, ${ }^{5}$ LEC in Sokoto-Zamfara resulted in improved cure rates among patients treated with MDT; and the adoption of mini-LECs in five neighbouring states. The authors recommend that LECs be maintained for at least 3 years, to accelerate leprosy elimination, since (i) LEC is cost effective in the region, (ii) repeat mini-LECs continue to yield cases, and (iii) annual new case detection figures of 1999 and 2000 remain higher than pre-LEC annual case detection of 1994, 1996 and 1998.

The authors agree with WHO that in assessing the performance of LECs, looking at case 
detection alone is insufficient. Attention should also to be given to improvement in MDT coverage, capacity building, increasing community awareness, and the review of cure rates, (which should be maintained at around $90-95 \%$ MDT completion) ${ }^{2}$.

\section{Acknowledgements}

The authors are grateful to all those involved in the campaign. We are also grateful to WHO and TLM for financial and logistic support respectively and to Dr N. Chitimba for technical guidance. We thank Professor W. C. S. Smith, Dr O. Ogbeiwi and Jannine Ebenso for useful comments.

\section{References}

1 World Health Organization. Weekly Epidemiological Record, 1999; 74: 329.

2 Report of a consultative meeting on leprosy elimination campaigns. WHO, Geneva, WHO/LEP/99.3.

${ }^{3}$ Leprosy elimination campaigns. Lepr Rev, 1999; 70: 430-494

${ }^{4}$ Leprosy elimination campaigns. Lepr Rev, 2000; 71: 62-80

5 Sofola O. Leprosy elimination campaigns: the Nigerian experience. Lepr Rev, 1999; 70: 465-471.

${ }^{6}$ World Health Organization. A guide to eliminating leprosy as public health problem, pocket edition. WHO, Geneva, 1997, p 61.

7 Smith W. C. S. Future scope and expectations: why, when, and how LECs should continue. Lepr Rev, 1999; 70, 498-505.

${ }^{8}$ Ganapati R et al. The Dharavi Story - saga of LECs over 2 decades. Lepr Rev, 1999; 70: 495-497.

9 World Health Organization. Weekly Epidemiological Record, 1999; 74, 333-334. 